\title{
DETERMINANTS OF G7 AND CHINESE STOCK MARKET RETURNS DURING COVID-19 OUTBREAK
}

\author{
Ahmed JERIBI \\ Faculty of Economics and Management of Mahdia, Mahdia, Tunisia \\ ahmedjeribi07@yahoo.fr \\ Mohamed FAKHFEKH \\ Higher Institute of Business Administration of Sfax, Sfax, Tunisia \\ fakhfekh_moh@yahoo.fr
}

\begin{abstract}
The purpose of this paper is to discuss the determinants of $G 7$, and Chinese stock market returns during the COVID-19 outbreak. We find that Bitcoin and Ethereum can generate benefits from portfolio diversification and hedging strategies for G7 financial investors in early 2020. Our result reveals that Gold is neither hedge nor haven during the COVID-19 pandemic. In addition, the results indicated that the expected volatility of the US stock market has no effect on the Japanese and Chinese financial markets. Finally, our results suggest that the growth rate of confirmed COVID-19 cases and deaths has an impact only on the US stock market.
\end{abstract}

Keywords: COVID-19 cases and deaths, Digital assets, Gold, G7 Stock markets, WTI.

\section{INTRODUCTION}

The beginning of 2020 has been marked by two crucial events. First, the world faces a problem of an unusual nature known as the Coronavirus pandemic that emerged out of China in December 2019. This pandemic has plundered and destabilized the global financial and economic system. The uncertainties caused by the evolution of this pandemic and its impact on the global financial and economic systems are gripping. In mid-February, when international investors began to fear that the epidemic would turn into a global pandemic, stock prices fell sharply. April 30, 2020, more than 3 million cases of Covid-19 as well as 230.000 deaths have been detected worldwide. The COVID-19 pandemic has caused a human and health crisis. April 14, 2020, the rapport of the International Monetary Fund indicated that the G7 countries had entered into a "deep recession". It declared that global growth which is projected at -3 percent in 2020 is "far worse" than during the Great Recession in 2009. Second, an oil price war between Russia and Saudi Arabia triggered in March 2020 has negative Future oil prices therefore, 
something that has never happened since NYMEX oil futures began trading in 1983 . Fueled by investors panic, global stock markets and crude oil price dived successively. During the crash, global stock markets become more volatile, mainly due to extreme uncertainty in the markets. The VIX index, as a measure of the expected volatility of the US stock market, became 82 on March 16, 2020, exceeding the spike we saw during the Financial Crisis of 2008. As a comparison, the average VIX for 2019 was 13.8. According to Goldman Sachs' report", the world economy will suffer its worst year since the Great Depression of the 1930s.

The rise in cross-market linkages or spillovers has raised portfolio riskiness and reduced diversification advantages, forcing investors and portfolio managers to find alternative assets that help to mitigate the risk exposure. For centuries, gold has traditionally been considered a safe haven during numerous crises (Baur and Lucey, 2010; Beckmann et al., 2015; Klein et al., 2018). Before the COVID-19 pandemic arrived on the scene, digital assets have become a slowly maturing niche. The last decade has seen a new kind of Bitcoin, first introduced by Nakamoto (2008). As with precious metals, Bitcoin has proved to be resistant in times of turmoil since it was founded in 2009, strengthening its potential position as a hedge and safe haven for financial and commodity markets against increasing global instability (Dyhrberg, 2016; Tiwari et al., 2019; Bouri et al, 2020). This has led to digital assets and gold has long been competing with each other for the status of a safe haven asset. Recently, in addition to the G7 countries, China became a dominant component of the global economy (He et al. 2014; Yu et al 2018). Based on this background, the impact of the global COVID-19 pandemic is explored by addressing this specific question: how cryptocurrencies, VIX, oil, and gold prices, as well as the number of COVID 19 cases and deaths, impact G7 and Chinese stock markets?

The rest of the paper is organized as follows. Section 2 presents methodology and data. Section 3 discusses the results. Section 4 concludes.

\section{METHODOLOGY AND DATA}

Amid worries over a deep recession of $\mathrm{G} 7$ economies with the propagation of the Coronavirus, we try to analyze the stock price responses to the increase of number of cases and deaths caused by the COVID-19 pandemic. In addition, we study the impact of the price variation of cryptocurrencies and Gold which are considered in many studies as safe-haven assets, as well as the VIX index and oil price war between Russia and Saudi Arabia on G7 and Chinese stock markets indices. To do this, we use the following regressions:

$$
\text { INDEX }_{t}=\alpha_{0}+\alpha_{1} \text { CRYPTO }_{t}+\alpha_{2} \text { VIX }_{t}+\alpha_{3} \text { WTI }_{t}+\alpha_{4} G O L D_{t}+\varepsilon_{t}(1)
$$

*Equity Research, Global Macro Research ISSUE 87 | March 24, 2020 


$$
\operatorname{INDEX}_{t}=\alpha_{0}+\alpha_{1} \text { CRYPTO }_{t}+\alpha_{2} \operatorname{VIX}_{t}+\alpha_{3} W_{t}+\alpha_{4} G O L D_{t}+\alpha_{5} C C_{t}+\alpha_{6} C D_{t}+\varepsilon_{t}(2)
$$

With:

INDEX ${ }_{t}:$ The studied index's daily return;

$V I X_{t}:$. The VIX index's daily return, as a measure of the expected volatility of the US stock market;

$W T I_{t}$ : The WTI crude oil's daily return;

$G O L D_{t}:$ The GOLD daily return;

$C C_{t}$ : The growth rate of confirmed COVID-19 cases;

$C D_{t}$ : The growth rate of confirmed COVID-19 deaths.

Daily time series data are collected for stock prices of the United States (S\&P500 and NASDAQ), France (CAC40), Germany (DAX30), the United Kingdom (FTSE 100), Italy (MIB 30), Japan (Nikkei 225), Canada (SP-TSX) and China (SSE) from DataStream covering the period from September 02 , 2019, to April 30, 2020. From the same database, we extracted the VIX index, WTI, and Gold prices. Data concerning the five studied cryptocurrencies (Bitcoin, Dash, Ethereum, Ripple, and Monero) was collected from the Coin Market Cap basis. The number of cases and deaths is collected from European Centre for Disease Prevention and Control (An agency of the European Union). The empirical study research involves 171 daily observations. In order to cover the effect of 2020 global pandemic, the research period is divided into two sub-periods. The first sub-period is called the period before COVID19 outbreak, goes from 02 September 2019 to 30 December 2019. The second one is the period before COVID-19 outbreak, and it goes from 31 December 2019 to 30 April 2020. Daily returns are defined by $r_{t}=\ln \left(p_{t} / p_{t-1}\right)$, with $P_{i, t}$ denoting indices I closing price at time t. In literature, this transformation of data is usually employed for series to obtain stationary data. In addition, figure 1 and 2 confirms that the return series are stationary. The entirety of the series was more volatile before the COVID-19 period than during the COVID-19 outbreak. Notably, all the series returns in figure 2 reached their negative peaks in late February and during March 2020.

\section{RESULTS}

The summary statistics of returns, including positive and negative returns as well as volatility before and during the COVID-19 periods, are given in Table 1. The results indicate that the highest mean of growth rates is for Italy's cumulative number of COVID-19 cases, followed by the USA's cumulative number of COVID cases. WTI prove the lowest mean followed by the MIB stock market index. Indeed, the results show that all stock market indices return have negative mean except for Nasdaq while the other series 
have a positive mean. The growth rate of COVID-19 cases in Italy has the highest standard deviation followed by China. An important result is found, all cryptocurrency returns became positive compared to those during the pre-corona virus period. Indeed, all stock market indices returns turns out to be lower or even negative than those in the pre-corona virus period. Furthermore, all series seem to be more volatile during the COVID-19 period than those before COVID-10 outbreak. All series have excess levels of kurtosis during the two periods, especially the growth rate of COVID-19 cases in Italy. All growth rates of COVID-19 cases and deaths as well as VIX, Dash, and Nikkei indices during the two periods and Bitcoin, Monero, Ripple and WTI during the pre-corona virus period have a positive skewness, whereas the rest have a positive one. Moreover, the Jarque-Bera tests also show that all the series are non-normal except for Nasdaq and SSE stock market indices during the pre-COVID period, thus stress the importance of tail characteristics (e.g. extreme risk "COVID-19 outbreak") of asset return distributions.
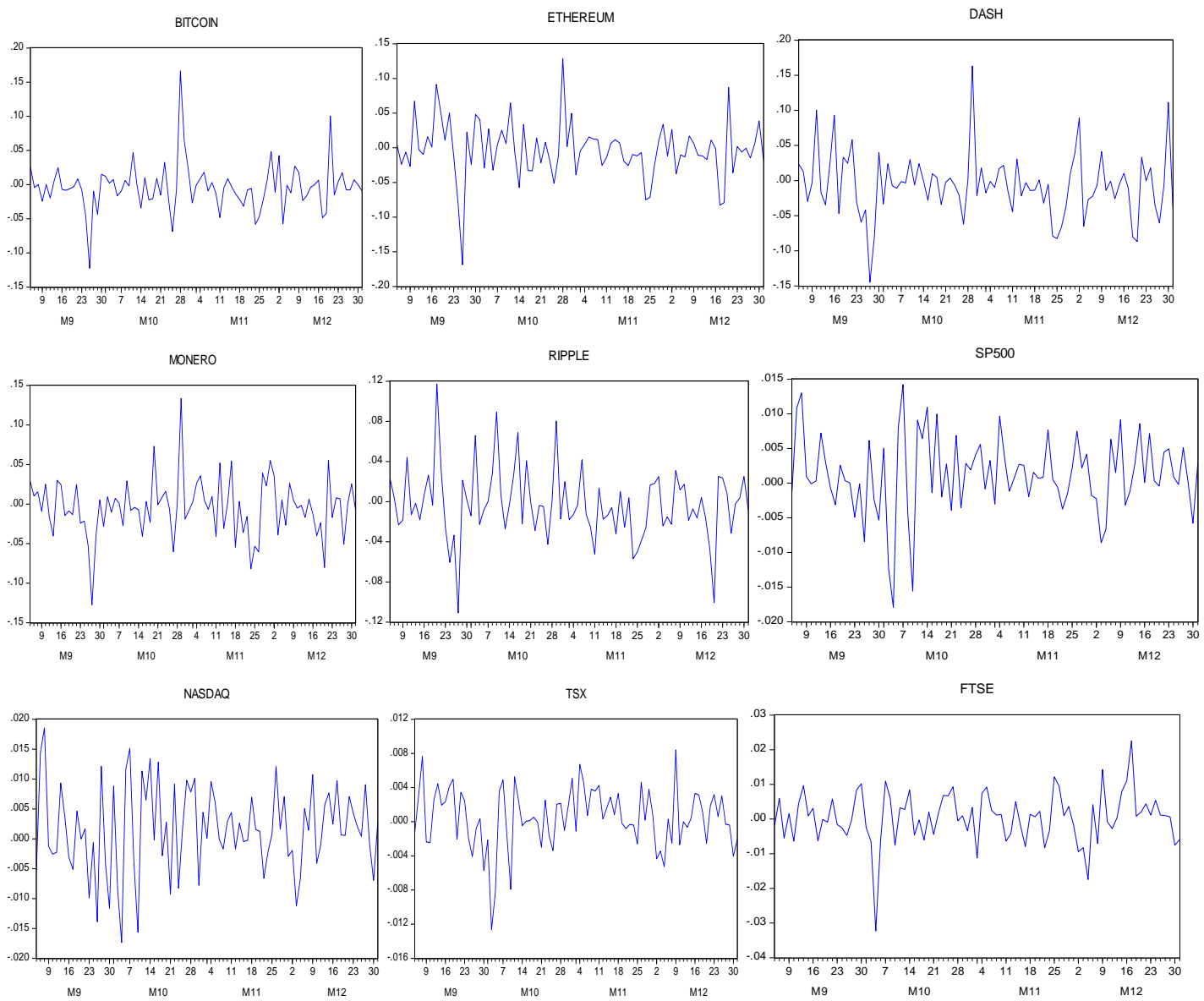
Jeribi, A., Fakhfekh, M.

DETERMINANTS OF G7 AND CHINESE STOCK MARKET RETURNS DURING COVID-19 OUTBREAK
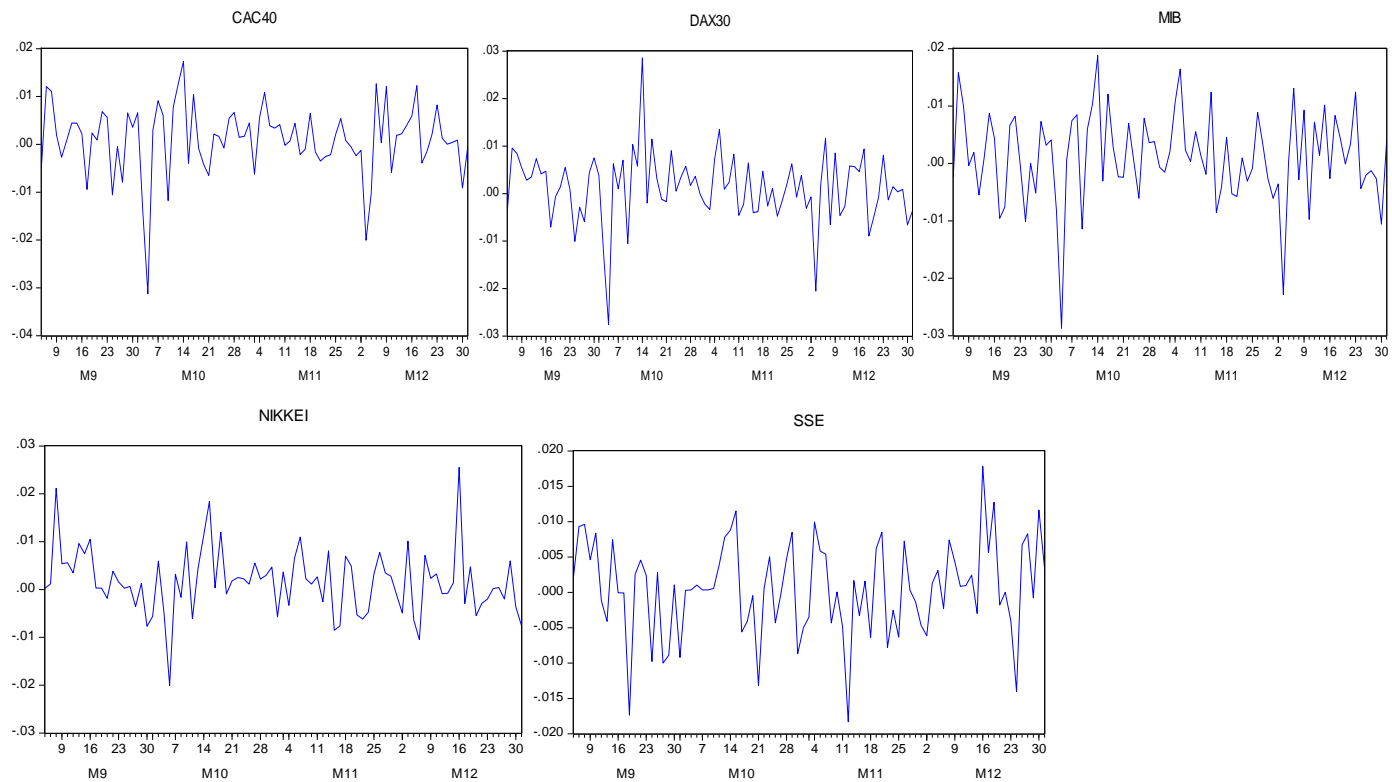

FIGURE 1: EVOLUTION OF SERIES RETURNS BEFORE COVID-19 OUTBREAK Source: authors
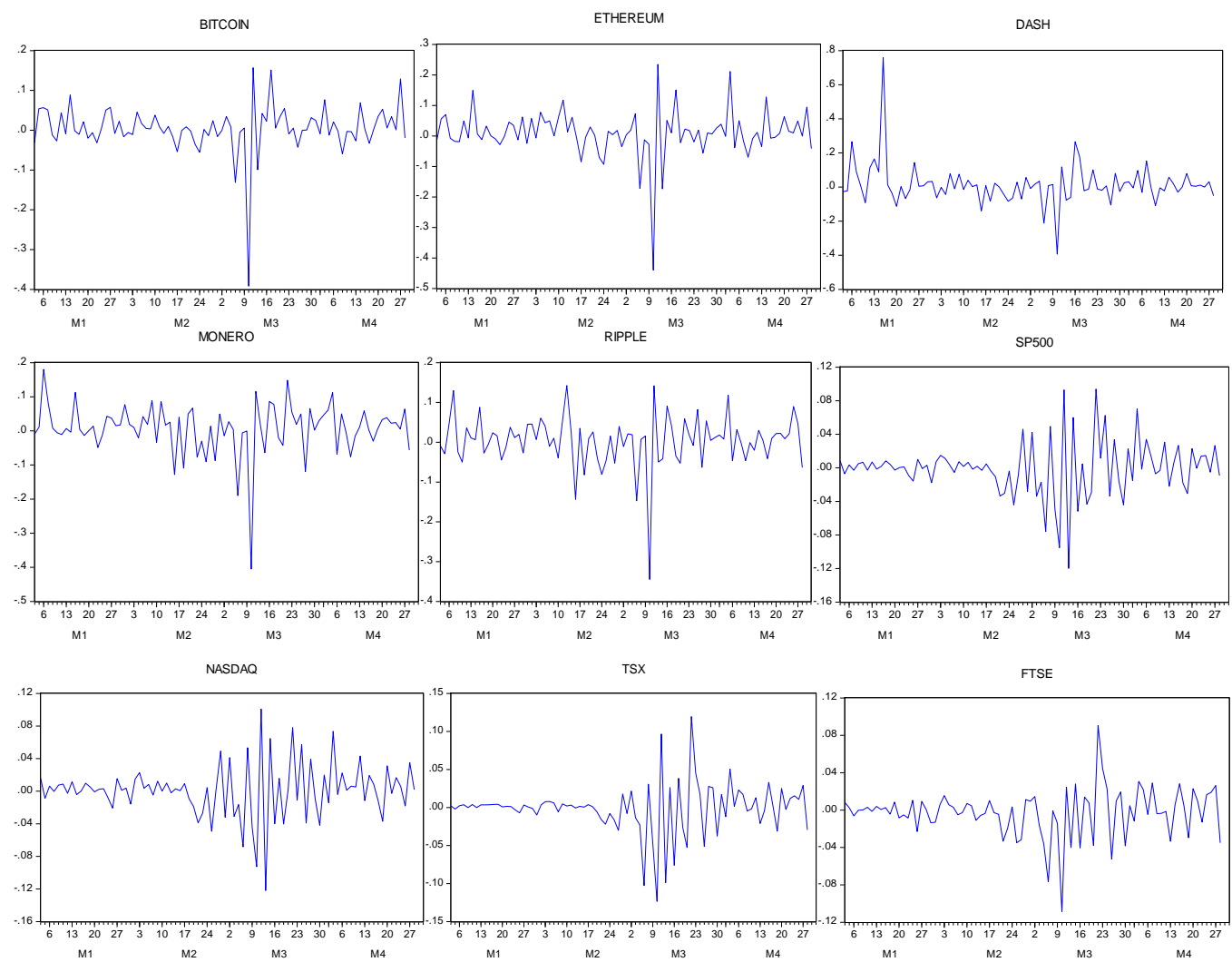

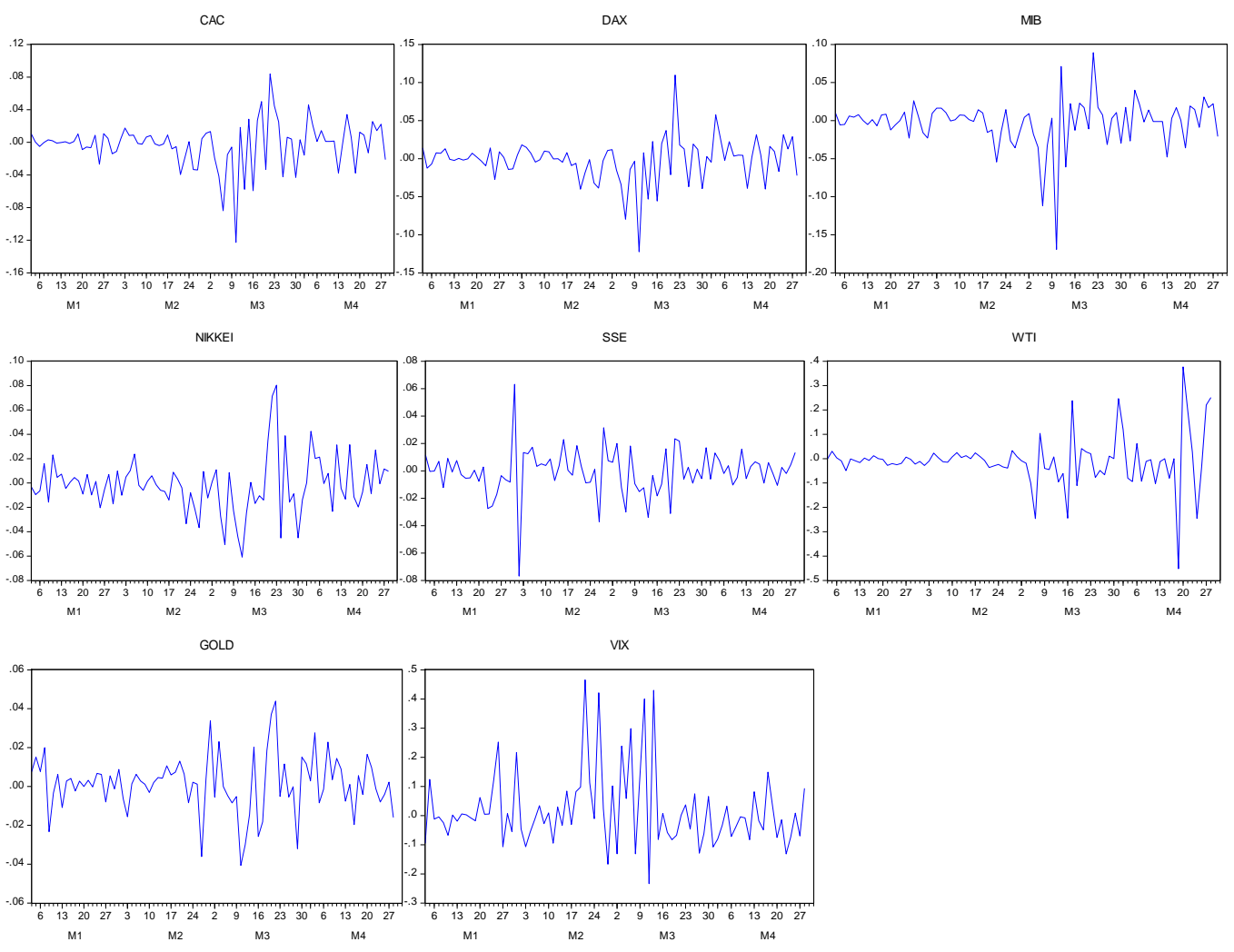

FIGURE 2: EVOLUTION OF SERIES RETURNS DURING COVID-19 OUTBREAK

Source: authors

TABLE 1. SUMMARY STATISTICS FOR RETURNS

\begin{tabular}{|c|c|c|c|c|c|c|c|c|c|}
\hline \multirow{11}{*}{ 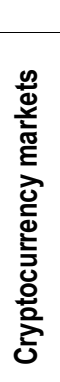 } & & Period & Mean & Max & Min & Std. Dev. & Skewness & Kurtosis & J-B \\
\hline & \multirow{2}{*}{ BITCOIN } & Before & $-0,0037$ & 0,1665 & $-0,1225$ & 0,0347 & 1,1699 & 10,4763 & $217,35^{\star \star \star}$ \\
\hline & & During & 0,0044 & 0,1571 & $-0,3918$ & 0,0620 & $-2,8414$ & 21,9316 & $1367,45^{\star * *}$ \\
\hline & \multirow{2}{*}{ DASH } & Before & $-0,0064$ & 0,1632 & $-0,1448$ & 0,0457 & 0,5674 & 5,4175 & $25,26^{* * *}$ \\
\hline & & During & 0,0142 & 0,7592 & $-0,3936$ & 0,1216 & 2,4746 & 19,5620 & $1045,78^{* * *}$ \\
\hline & \multirow{2}{*}{ ETHEREUM } & Before & $-0,0029$ & 0,1289 & $-0,1687$ & 0,0410 & $-0,3078$ & 6,2833 & $39,52^{* * *}$ \\
\hline & & During & 0,0092 & 0,2345 & $-0,4400$ & 0,0801 & $-1,7849$ & 14,4316 & $501,98^{* * *}$ \\
\hline & \multirow{2}{*}{ MONERO } & Before & $-0,0046$ & 0,1335 & $-0,1275$ & 0,0366 & 0,1265 & 5,4986 & $22,34^{* * *}$ \\
\hline & & During & 0,0069 & 0,1803 & $-0,4052$ & 0,0755 & $-2,0345$ & 12,5739 & $378,76^{* * *}$ \\
\hline & \multirow{2}{*}{ RIPPLE } & Before & $-0,0026$ & 0,1172 & $-0,1109$ & 0,0354 & 0,2988 & 5,1103 & $17,04^{\star \star \star}$ \\
\hline & & During & 0,0034 & 0,1427 & $-0,3446$ & 0,0653 & $-1,7249$ & 11,6244 & $301,98^{\star * *}$ \\
\hline & \multirow{2}{*}{ GOLD } & Before & 0,0000 & 0,0150 & $-0,0212$ & 0,0069 & $-0,5638$ & 3,4692 & $5,28^{*}$ \\
\hline & & During & 0,0014 & 0,0439 & $-0,0407$ & 0,0147 & $-0,1481$ & 4,2956 & $6,18^{* \star *}$ \\
\hline & \multirow{2}{*}{ WTI } & Before & 0,0016 & 0,1468 & $-0,0566$ & 0,0231 & 2,8349 & 19,8814 & $1123,16^{* * *}$ \\
\hline & & During & $-0,0080$ & 0,3766 & $-0,4521$ & 0,1060 & $-0,0348$ & 8,5091 & $106,24^{* * *}$ \\
\hline & \multirow{2}{*}{ VIX } & Before & $-0,0019$ & 0,1815 & $-0,1185$ & 0,0639 & 0,6647 & 3,3114 & $6,60^{* *}$ \\
\hline & & During & 0,0183 & 0,4655 & $-0,2337$ & 0,1305 & 1,5644 & 5,8722 & $63,14^{\star \star *}$ \\
\hline \multirow{9}{*}{ 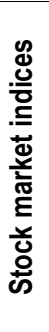 } & \multirow{2}{*}{ S\&P 500} & Before & 0,0012 & 0,0142 & $-0,0179$ & 0,0057 & $-0,5254$ & 4,2905 & $9,81^{* * *}$ \\
\hline & & During & $-0,0007$ & 0,0938 & $-0,1198$ & 0,0335 & $-0,2214$ & 5,5459 & $23,37^{\star \star \star}$ \\
\hline & \multirow{2}{*}{ NASDAQ } & Before & 0,0016 & 0,0186 & $-0,0174$ & 0,0073 & $-0,1896$ & 2,8242 & 0,62 \\
\hline & & During & 0,0009 & 0,1007 & $-0,1219$ & 0,0335 & $-0,2927$ & 5,5499 & $23,96^{\star \star \star}$ \\
\hline & \multirow{2}{*}{ FTSE 100} & Before & 0,0004 & 0,0225 & $-0,0323$ & 0,0074 & $-0,7552$ & 6,9249 & $62,64^{\star \star *}$ \\
\hline & & During & $-0,0026$ & 0,0905 & $-0,1087$ & 0,0259 & $-0,6738$ & 7,2003 & $68,10^{\star \star *}$ \\
\hline & \multirow{2}{*}{ NIKKEI 225} & Before & 0,0016 & 0,0255 & $-0,0201$ & 0,0068 & 0,4791 & 5,1729 & $19,97^{\star * *}$ \\
\hline & & During & $-0,0016$ & 0,0804 & $-0,0608$ & 0,0229 & 0,5830 & 5,2637 & $22,69^{\star * *}$ \\
\hline & DAX 30 & Before & 0,0012 & 0,0286 & $-0,0276$ & 0,0074 & $-0,4288$ & 6,8110 & $54,04^{\star \star *}$ \\
\hline
\end{tabular}


Jeribi, A., Fakhfekh, M.

DETERMINANTS OF G7 AND CHINESE STOCK MARKET RETURNS DURING COVID-19 OUTBREAK

\begin{tabular}{|c|c|c|c|c|c|c|c|c|c|}
\hline & & During & $-0,0019$ & 0,1098 & $-0,1224$ & 0,0284 & $-0,5332$ & 8,4382 & $107,49^{* * *}$ \\
\hline & $C \Delta C \Delta 0$ & Before & 0,0010 & 0,0173 & $-0,0312$ & 0,0074 & $-1,1682$ & 6,6674 & $66,97^{\star \star *}$ \\
\hline & CAC 40 & During & $-0,0028$ & 0,0839 & $-0,1228$ & 0,0281 & $-0,9702$ & 7,1312 & $72,91^{\text {*** }}$ \\
\hline & & Before & 0,0012 & 0,0188 & $-0,0287$ & 0,0079 & $-0,6782$ & 4,9126 & $19,47^{\star \star \star}$ \\
\hline & IVIID & During & $-0,0029$ & 0,0893 & $-0,1692$ & 0,0318 & $-1,9063$ & 12,5178 & $367,94^{\star \star \star}$ \\
\hline & TSX & Before & 0,0005 & 0,0084 & $-0,0127$ & 0,0035 & $-0,6699$ & 4,4490 & $13,79^{* * *}$ \\
\hline & $15 x$ & During & $-0,0011$ & 0,1196 & $-0,1235$ & 0,0338 & $-0,4768$ & 7,4725 & $73,19^{* \star *}$ \\
\hline & & Before & 0,0005 & 0,0178 & $-0,0183$ & 0,0067 & $-0,3372$ & 3,3429 & 2,03 \\
\hline & SSE & During & $-0,0006$ & 0,0632 & $-0,0768$ & 0,0171 & $-0,6074$ & 8,3722 & $106,18^{\star \star \star}$ \\
\hline & CCC & & 0,1556 & 1,2029 & 0,0000 & 0,2279 & 2,3748 & 9,3738 & $221,14^{\star \star \star}$ \\
\hline$\frac{\text { C }}{\text { ত }}$ & CDC & & 0,1322 & 3,0000 & 0,0000 & 0,3794 & 5,6931 & 40,9074 & $5483,17^{\star \star \star \star}$ \\
\hline g & $\mathrm{CCJ}$ & & 0,1354 & 1,0000 & 0,0000 & 0,2069 & 2,3726 & 8,8183 & $197,30^{* * *}$ \\
\hline J & CDJ & & 0,0915 & 2,0000 & 0,0000 & 0,2545 & 5,7364 & 40,7147 & $5439,09^{* * *}$ \\
\hline$\stackrel{9}{\square}$ & CCG & & 0,1994 & 3,0000 & 0,0000 & 0,4414 & 4,0618 & 22,6585 & $1583,57^{\star * *}$ \\
\hline 을 & $C D G$ & & 0,1302 & 2,3077 & 0,0000 & 0,3382 & 4,3808 & 24,8342 & $1937,24^{\star \star \star}$ \\
\hline & $\mathrm{CCl}$ & & 0,6204 & 43,0000 & 0,0000 & 4,6857 & 8,9659 & 81,5939 & $22744,95^{\star \star \star}$ \\
\hline & CDI & During & 0,1497 & 2,0000 & 0,0000 & 0,3244 & 3,5777 & 17,5883 & $924,07^{\star * \star}$ \\
\hline & CCF & & 0,1692 & 2,4211 & 0,0000 & 0,3651 & 4,1102 & 22,3036 & $1540,72^{\star \star \star}$ \\
\hline & CDF & & 0,1544 & 1,7143 & 0,0000 & 0,2946 & 2,8728 & 12,5939 & $437,69^{\star * *}$ \\
\hline 0 & $\mathrm{CCCH}$ & & 0,1581 & 5,1270 & 0,0000 & 0,6134 & 6,8675 & 53,8593 & $9713,61^{* \star \star}$ \\
\hline & $\mathrm{CDCH}$ & & 0,1440 & 2,1154 & 0,0000 & 0,3931 & 3,9137 & 18,2781 & $1031,41^{* * *}$ \\
\hline & CCUSA & & 0,2286 & 4,0000 & $-1,0000$ & 0,5432 & 4,4882 & 30,6638 & $2854,79^{* * *}$ \\
\hline 疍 & CDUSA & & 0,1551 & 2,1400 & $-1,0000$ & 0,3813 & 3,0487 & 17,7067 & $855,44^{* * *}$ \\
\hline (5 & CCUK & & 0,1709 & 1,3739 & 0,0000 & 0,2938 & 2,6582 & 10,1309 & $276,90^{\star * \star}$ \\
\hline & CDUK & & 0,1684 & 2,5000 & 0,0000 & 0,3987 & 3,9368 & 20,4491 & $1282,62^{* * *}$ \\
\hline
\end{tabular}

Note: CC indicate the growth rate of confirmed COVID-19 cases while CD indicatethe growth rate of confirmed COVID-19 deaths. C, J, G, I, F, CH, USA and UK design the name of countries "Canada, Japan, Germany, Italy, France, China, USA and UK", respectiverly. *** "** and" means significant at $1 \%, 5 \%$ and $10 \%$ level of significance respectively. Where St.dev represents the standard deviation of returns and JB Stat indicates the Jarque-Bera statistics.

We empirically study the effect of cryptocurrencies, VIX, oil, and gold prices as well as the growth rates of COVID 19 cases and deaths on G7 and Chinese stock. The results of the OLS regression are given in Table 2 and 3.

TABLE 2. OLS REGRESSION RESULT ESTIMATION BEFORE COVID-19 OUTBREAK

\begin{tabular}{|c|c|c|c|c|c|c|c|c|c|c|}
\hline & $C$ & Bitcoin & Ethereum & Dash & Monero & Ripple & VIX & WTI & GOLD & $\mathbf{R}^{2}$ \\
\hline \multirow{5}{*}{$\begin{array}{l}\text { 吕 } \\
\text { के }\end{array}$} & $0,001^{* * *}$ & 0,007 & - & - & - & - & $-0,066^{\star \star *}$ & 0,010 & $-0,168^{* \star *}$ & 0,694 \\
\hline & $0,001^{* * *}$ & - & $-0,006$ & - & - & - & $-0,067^{* \star *}$ & 0,010 & $-0,169^{* \star *}$ & 0,694 \\
\hline & $0,001^{* * *}$ & - & - & 0,003 & - & - & $-0,067^{* \star *}$ & 0,010 & $-0,166^{\star \star *}$ & 0,693 \\
\hline & $0,001^{* * *}$ & - & - & - & $0,017^{*}$ & - & $-0,068^{* * *}$ & 0,010 & $-0,171^{* * *}$ & 0,704 \\
\hline & $0,001^{* * *}$ & - & - & - & - & 0,0002 & $-0,067^{\star * \star}$ & 0,009 & $-0,167^{\star * *}$ & 0,692 \\
\hline \multirow{5}{*}{ 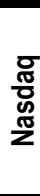 } & $0,002^{\star \star \star}$ & 0,023 & - & - & - & - & $-0,080^{\star * *}$ & $-0,005$ & $-0,188^{* *}$ & 0,612 \\
\hline & $0,001^{* * *}$ & - & 0,0001 & - & - & - & $-0,081^{* * *}$ & $-0,008$ & $-0,183^{* *}$ & 0,601 \\
\hline & $0,001^{* * *}$ & - & - & $-0,002$ & - & - & $-0,081^{* \star *}$ & $-0,009$ & $-0,183^{* *}$ & 0,601 \\
\hline & $0,002^{* * *}$ & - & - & - & 0,016 & - & $-0,082^{\star \star \star}$ & $-0,008$ & $-0,187^{* *}$ & 0,607 \\
\hline & $0,001^{* * *}$ & - & - & - & - & 0,002 & $-0,082^{\star \star *}$ & $-0,008$ & $-0,183^{* *}$ & 0,601 \\
\hline \multirow{5}{*}{ 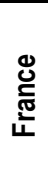 } & 0,001 & 0,005 & - & - & - & - & $-0,068^{\star * *}$ & 0,003 & $-0,202^{* *}$ & 0,459 \\
\hline & 0,001 & - & $-0,011$ & - & - & - & $-0,069^{* * *}$ & 0,003 & $-0,205^{\star *}$ & 0,462 \\
\hline & $0,001^{*}$ & - & - & $0,027^{* *}$ & - & - & $-0,071^{* \star *}$ & 0,009 & $-0,196^{* *}$ & 0,485 \\
\hline & 0,001 & - & - & - & 0,022 & - & $-0,070^{\star \star \star}$ & 0,003 & $-0,206^{* *}$ & 0,470 \\
\hline & 0,001 & - & - & - & - & 0,027 & $-0,071^{* \star *}$ & 0,003 & $-0,205^{* *}$ & 0,475 \\
\hline
\end{tabular}




\begin{tabular}{|c|c|c|c|c|c|c|c|c|c|c|}
\hline \multirow{5}{*}{ 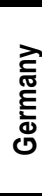 } & 0,001 & 0,006 & - & - & - & - & $-0,059^{* \star \star}$ & 0,009 & $-0,192^{*}$ & 0,360 \\
\hline & 0,001 & - & $-0,009$ & - & - & - & $-0,060^{* * *}$ & 0,009 & $-0,195^{\star}$ & 0,362 \\
\hline & $0,001^{*}$ & - & - & $0,031^{* *}$ & - & - & $-0,062^{* \star *}$ & 0,016 & $-0,186^{*}$ & 0,396 \\
\hline & $0,001^{*}$ & - & - & - & 0,026 & - & $-0,061^{* \star *}$ & 0,008 & $-0,198^{* *}$ & 0,375 \\
\hline & $0,001^{*}$ & - & - & - & - & 0,031 & $-0,063^{\star \star \star}$ & 0,009 & $-0,196^{*}$ & 0,380 \\
\hline \multirow{5}{*}{ 可 } & 0,001 & 0,013 & - & - & - & - & $-0,063^{* * *}$ & 0,024 & $-0,226^{* *}$ & 0,377 \\
\hline & 0,001 & - & $-0,015$ & - & - & - & $-0,064^{* \star *}$ & 0,024 & $-0,230^{* *}$ & 0,380 \\
\hline & 0,001 & - & - & 0,022 & - & - & $-0,065^{\star * *}$ & 0,028 & $-0,220^{* \star}$ & 0,390 \\
\hline & 0,001 & - & - & - & 0,026 & - & $-0,065^{* * *}$ & 0,023 & $-0,231^{* \star}$ & 0,388 \\
\hline & 0,001 & - & - & - & - & 0,027 & $-0,066^{\star * *}$ & 0,023 & $-0,228^{* *}$ & 0,388 \\
\hline \multirow{5}{*}{ 弚 } & 0,000 & $-0,017$ & - & - & - & - & $-0,048^{* * *}$ & 0,014 & $-0,150$ & 0,232 \\
\hline & 0,000 & - & $-0,027$ & - & - & - & $-0,047^{\star * *}$ & 0,020 & $-0,164$ & 0,248 \\
\hline & 0,000 & - & - & 0,000 & - & - & $-0,047^{* * *}$ & 0,017 & $-0,154$ & 0,226 \\
\hline & 0,000 & - & - & - & $-0,010$ & - & $-0,046^{\star \star \star}$ & 0,016 & $-0,151$ & 0,229 \\
\hline & 0,000 & - & - & - & - & 0,007 & $-0,048^{* * *}$ & 0,017 & $-0,155$ & 0,227 \\
\hline \multirow{5}{*}{$\begin{array}{l}\text { శ్ } \\
\text { శ్ } \\
\text { శ్ }\end{array}$} & $0,002^{* *}$ & $-0,004$ & - & - & - & - & 0,000 & 0,009 & $-0,146$ & 0,024 \\
\hline & $0,002^{* *}$ & - & $-0,005$ & - & - & - & 0,000 & 0,010 & $-0,149$ & 0,024 \\
\hline & $0,002^{* *}$ & - & - & 0,008 & - & - & $-0,001$ & 0,011 & $-0,145$ & 0,026 \\
\hline & $0,002^{* *}$ & - & - & - & 0,002 & - & 0,000 & 0,009 & $-0,147$ & 0,024 \\
\hline & $0,002^{* *}$ & - & - & - & - & 0,022 & $-0,002$ & 0,010 & $-0,150$ & 0,036 \\
\hline \multirow{5}{*}{$\begin{array}{l}\frac{\pi}{\pi} \\
\text { त్ } \\
\widetilde{J}\end{array}$} & 0,000 & 0,005 & - & - & - & - & $-0,029^{\star * *}$ & 0,023 & $-0,038$ & 0,349 \\
\hline & 0,000 & - & 0,007 & - & - & - & $-0,030^{* * *}$ & 0,021 & $-0,035$ & 0,353 \\
\hline & 0,000 & - & - & 0,002 & - & - & $-0,030^{* * *}$ & 0,022 & $-0,037$ & 0,347 \\
\hline & 0,000 & - & - & - & 0,007 & - & $-0,030^{\star \star \star}$ & 0,022 & $-0,039$ & 0,352 \\
\hline & 0,000 & - & - & - & - & $-0,008$ & $-0,029^{\star \star \star}$ & 0,022 & $-0,036$ & 0,353 \\
\hline \multirow{5}{*}{ : } & 0,000 & 0,022 & - & - & - & - & $-0,006$ & $0,061^{*}$ & 0,044 & 0,060 \\
\hline & 0,000 & - & $-0,001$ & - & - & - & $-0,008$ & $0,058^{*}$ & 0,048 & 0,047 \\
\hline & 0,001 & - & - & $0,027^{*}$ & - & - & $-0,010$ & $0,065^{\star *}$ & 0,053 & 0,079 \\
\hline & 0,001 & - & - & - & 0,031 & - & $-0,010$ & $0,059^{*}$ & 0,040 & 0,074 \\
\hline & 0,000 & - & - & - & - & 0,028 & $-0,010$ & $0,059^{*}$ & 0,045 & 0,068 \\
\hline
\end{tabular}

Note: CC indicate cumulative number of COVID-19 cases while CD indicate cumulative number of COVID-19 deaths. ${ }^{* * *}$, ${ }^{* *}$ and ${ }^{*}$ means significant at $1 \%, 5 \%$ and $10 \%$ level of significance respectively.

TABLE 3. OLS REGRESSION RESULT ESTIMATION DURING COVID-19 OUTBREAK

\begin{tabular}{|c|c|c|c|c|c|c|c|c|c|c|c|c|}
\hline & C & Bitcoin & Ethereum & Dash & Monero & Ripple & VIX & WTI & GOLD & $\mathrm{CC}$ & $C D$ & $\mathbf{R}^{2}$ \\
\hline \multirow{5}{*}{ 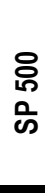 } & 0,003 & $0,098^{*}$ & - & - & - & - & $-0,161^{* \star *}$ & 0,022 & $0,399^{* *}$ & 0,003 & $-0,014^{*}$ & 0,609 \\
\hline & 0,002 & - & $0,101^{* \star *}$ & - & - & - & $-0,152^{\star * *}$ & 0,022 & $0,384^{* *}$ & 0,002 & $-0,013^{* *}$ & 0,627 \\
\hline & 0,004 & - & & $-0,001$ & - & - & $-0,185^{\star \star *}$ & 0,027 & $0,524^{* * *}$ & 0,005 & $-0,017^{* *}$ & 0,589 \\
\hline & 0,003 & - & - & - & 0,028 & - & $-0,180^{* * *}$ & 0,026 & $0,491^{* *}$ & 0,004 & $-0,016^{* *}$ & 0,592 \\
\hline & 0,003 & - & - & - & - & 0,026 & $-0,180^{\star \star \star}$ & 0,027 & $0,505^{\star \star \star}$ & 0,004 & $-0,016^{* *}$ & 0,591 \\
\hline \multirow{5}{*}{$\begin{array}{l}\bar{g} \\
\frac{\pi}{0} \\
\frac{\pi}{2} \\
\end{array}$} & 0,004 & $0,088^{*}$ & - & - & - & - & $-0,175^{\star \star \star}$ & 0,029 & $0,309^{*}$ & 0,004 & $-0,008$ & 0,651 \\
\hline & 0,003 & - & $0,098^{* \star *}$ & & - & - & $-0,165^{* * *}$ & 0,028 & $0,285^{*}$ & 0,004 & $-0,007$ & 0,671 \\
\hline & 0,004 & - & - & $-0,006$ & - & - & $-0,198^{* * *}$ & 0,033 & $0,424^{* *}$ & 0,006 & $-0,011$ & 0,635 \\
\hline & 0,004 & - & - & - & 0,014 & - & $-0,194^{* \star *}$ & 0,033 & $0,403^{* \star}$ & 0,006 & $-0,010$ & 0,636 \\
\hline & 0,004 & - & - & - & - & 0,010 & $-0,194^{* * *}$ & 0,033 & $0,413^{* *}$ & 0,006 & $-0,010$ & 0,635 \\
\hline \multirow{5}{*}{ 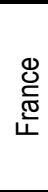 } & 0,000 & $0,123^{\star * *}$ & - & - & - & - & $-0,090^{* * *}$ & 0,005 & $0,510^{\star * *}$ & $-0,010$ & $-0,006$ & 0,561 \\
\hline & 0,000 & - & $0,074^{* *}$ & - & - & - & $-0,093^{\star \star *}$ & 0,009 & $0,562^{* * *}$ & $-0,009$ & $-0,007$ & 0,541 \\
\hline & 0,001 & - & - & 0,014 & & - & $-0,112^{* * *}$ & 0,013 & $0,638^{* \star *}$ & $-0,010$ & $-0,007$ & 0,514 \\
\hline & 0,000 & - & - & - & $0,087^{\star \star *}$ & - & $-0,103^{* \star *}$ & 0,013 & $0,562^{\text {***}}$ & $-0,009$ & $-0,004$ & 0,556 \\
\hline & 0,000 & - & - & - & - & $0,077^{* *}$ & $-0,104^{* * *}$ & 0,016 & $0,607^{* * *}$ & $-0,009$ & $-0,005$ & 0,537 \\
\hline \multirow{5}{*}{ 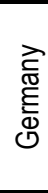 } & $-0,001$ & $0,121^{* \star *}$ & - & - & - & - & $-0,095^{\star \star \star}$ & 0,007 & $0,606^{* * *}$ & $-0,007$ & 0,005 & 0,559 \\
\hline & $-0,001$ & - & $0,075^{\star *}$ & & - & - & $-0,098^{* * *}$ & 0,012 & $0,650^{* * *}$ & $-0,007$ & 0,005 & 0,543 \\
\hline & 0,000 & - & - & 0,019 & - & - & $-0,116^{* * *}$ & 0,015 & $0,732^{\text {*** }}$ & $-0,007$ & 0,003 & 0,519 \\
\hline & $-0,001$ & - & - & - & $0,100^{* \star *}$ & - & $-0,103^{* * *}$ & 0,014 & $0,644^{\star * *}$ & $-0,006$ & 0,005 & 0,573 \\
\hline & $-0,001$ & - & - & - & & $0,101^{* * *}$ & $-0,103^{\star \star \star}$ & 0,018 & $0,685^{\star \star *}$ & $-0,007$ & 0,006 & 0,558 \\
\hline
\end{tabular}




\begin{tabular}{|c|c|c|c|c|c|c|c|c|c|c|c|c|}
\hline \multirow{5}{*}{ 촐 } & $-0,001$ & $0,243^{* * *}$ & - & - & - & - & $-0,091^{* \star *}$ & $-0,002$ & $0,350^{* * *}$ & 0,000 & $-0,010$ & 0,672 \\
\hline & $-0,002$ & - & $0,153^{\star \star *}$ & - & - & - & $-0,098^{* \star *}$ & 0,008 & $0,439^{* \star *}$ & 0,000 & $-0,009$ & 0,622 \\
\hline & 0,000 & - & - & $0,041^{*}$ & - & - & $-0,137^{\star \star *}$ & 0,013 & $0,583^{* * *}$ & 0,000 & $-0,013^{*}$ & 0,551 \\
\hline & $-0,001$ & - & - & - & $0,158^{* * *}$ & - & $-0,118^{* \star *}$ & 0,013 & $0,471^{* * *}$ & 0,000 & $-0,006$ & 0,637 \\
\hline & $-0,001$ & - & - & - & - & $0,170^{* \star *}$ & $-0,116^{\star * \star}$ & 0,019 & $0,533^{* * *}$ & 0,000 & $-0,008$ & 0,624 \\
\hline \multirow{5}{*}{ 亭 } & 0,000 & $0,124^{\star \star *}$ & - & - & - & - & $-0,080^{\star \star \star}$ & 0,008 & $0,405^{\star \star \star}$ & $-0,011$ & 0,001 & 0,521 \\
\hline & $-0,001$ & - & $0,072^{\star \star}$ & - & - & - & $-0,085^{\star \star \star}$ & 0,014 & $0,458^{\star \star \star}$ & $-0,011$ & 0,002 & 0,494 \\
\hline & 0,000 & - & - & 0,027 & - & - & $-0,101^{* * *}$ & 0,018 & $0,526^{* * *}$ & $-0,010$ & 0,001 & 0,475 \\
\hline & $-0,001$ & - & - & - & $0,103^{* * *}$ & & $-0,091^{* \star *}$ & 0,017 & $0,442^{* * *}$ & $-0,006$ & 0,000 & 0,537 \\
\hline & $-0,001$ & - & - & - & - & $0,094^{* * *}$ & $-0,092^{\star \star \star}$ & 0,020 & $0,494^{* \star *}$ & $-0,007$ & 0,001 & 0,507 \\
\hline \multirow{5}{*}{ 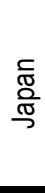 } & 0,000 & 0,002 & - & - & - & - & $-0,026$ & 0,014 & $0,524^{* \star \star}$ & $-0,011$ & $-0,006$ & 0,173 \\
\hline & 0,000 & - & $-0,010$ & & - & - & $-0,030$ & 0,014 & $0,538^{* * \star}$ & $-0,010$ & $-0,005$ & 0,174 \\
\hline & 0,000 & - & - & 0,007 & - & - & $-0,026$ & 0,014 & $0,521^{* * *}$ & $-0,010$ & $-0,005$ & 0,174 \\
\hline & $-0,001$ & - & - & - & 0,051 & - & $-0,021$ & 0,012 & $0,472^{* \star *}$ & $-0,007$ & $-0,002$ & 0,196 \\
\hline & 0,000 & - & - & - & - & 0,043 & $-0,021$ & 0,014 & $0,501^{* \star \star}$ & $-0,009$ & $-0,004$ & 0,186 \\
\hline \multirow{5}{*}{$\begin{array}{l}\frac{\pi}{0} \\
\stackrel{0}{\pi} \\
\mathbb{\pi} \\
0\end{array}$} & 0,003 & $0,213^{* \star *}$ & - & - & - & - & $-0,101^{* * *}$ & 0,033 & 0,247 & $-0,021^{*}$ & $-0,001$ & 0,564 \\
\hline & 0,002 & - & $0,165^{\star \star *}$ & - & - & - & $-0,097^{\star \star *}$ & 0,040 & 0,292 & $-0,018$ & $-0,002$ & 0,566 \\
\hline & 0,004 & - & - & 0,030 & - & - & $-0,138^{* \star *}$ & $0,050^{*}$ & $0,459^{* *}$ & $-0,019$ & 0,000 & 0,471 \\
\hline & 0,003 & - & - & - & $0,121^{* \star *}$ & - & $-0,123^{\star * *}$ & $0,049^{*}$ & $0,345^{*}$ & $-0,018$ & 0,003 & 0,522 \\
\hline & 0,003 & - & - & - & & $0,126^{* \star *}$ & $-0,122^{\star \star \star}$ & $0,054^{* *}$ & $0,401^{* *}$ & $-0,017$ & 0,002 & 0,510 \\
\hline \multirow{5}{*}{ : } & 0,000 & 0,005 & - & - & - & - & $-0,006$ & 0,024 & $0,320^{* *}$ & $-0,002$ & $-0,003$ & 0,142 \\
\hline & 0,000 & - & 0,001 & - & - & - & $-0,006$ & 0,025 & $0,324^{* *}$ & $-0,002$ & $-0,003$ & 0,142 \\
\hline & 0,000 & - & - & 0,007 & - & - & $-0,005$ & 0,025 & $0,321^{* *}$ & $-0,002$ & $-0,004$ & 0,144 \\
\hline & 0,000 & - & - & - & 0,025 & - & $-0,002$ & 0,024 & $0,301^{* *}$ & $-0,002$ & $-0,004$ & 0,152 \\
\hline & 0,000 & - & - & - & - & 0,019 & $-0,003$ & 0,025 & $0,315^{* *}$ & $-0,002$ & $-0,004$ & 0,146 \\
\hline
\end{tabular}

Note: CC indicate cumulative number of COVID-19 cases while CD indicate cumulative number of COVID-19 deaths. ***, ** and* means significant at $1 \%, 5 \%$ and $10 \%$ level of significance respectively.

The results of the regressions analyses indicate that there is a low significant positive relationship between Bitcoin and all G7 stock market indices except that of Japan during COVID-19 outbreak. It reveals that cryptocurrencies are rather isolated from the other markets. This result is similar to that of Corbet et al. (2018), Corbet et al. (2019), Aslanidis et al. (2019), Tiwari et al. (2019), and Charfeddine et al. (2020). The lower positive coefficient of Bitcoin suggested significant benefits for portfolio diversification and risk management when Bitcoin was added especially for US investors. This result is consistent with Brière et al. (2015), Bouri et al. (2017), Kajtazi and Moro (2018), Guesmi et al. (2019), and Charfeddine et al. (2020). The results suggest that Ethereum is more capable than Bitcoin to generate benefits from portfolio diversification and hedging strategies for all G7 investors except that of Japan. Monero and Ripple can be considered as diversifier assets for French, Italian, German, and English financial investors during the COVID-19 outbreak. However, Dash act as a diversifier asset for only Italian investors. Nevertheless, the results of the regressions analyses indicate that there is a low significant positive relationship between Dash and the CAC 40 and DAX 30. The same relationship is observed between Monero and SP 500

The results indicated that VIX negatively influences all G7 stock market indices except that of Japan before and during the Corona-virus pandemic. The expected volatility of the US stock market has no effect on 
the Japanese and Chinese financial markets. Furthermore, there is a positive and statistically relationship between the Canadian stock market indexes. Before the 2020 global pandemic, the relationship between Gold and US, French and Italian stock indices is negative. However, the results of the regressions analyses show that there is a significant positive relationship between Gold and all studied indices returns during this pandemic. The higher positive coefficient of Gold indicates that the yellow metal is neither hedge nor safe haven during the COVID-19 pandemic. This result is inconsistent with Baur and Lucy (2010) and Beckmann et al. (2015). Contrarily to the US stock market, the German stock market is the most influenced by variation in gold prices. Finally, our results show that the growth rate of confirmed COVID-19 cases has no impact on stock market returns. However, the growth rate of confirmed COVID-19 deaths influenced only the US stock market. In fact, the number of confirmed COVID-19 deaths in the USA represents more than $30 \%$ of the confirmed COVID-19 deaths in the entire world.

\section{CONCLUSION}

Given the massive impacts of the coronavirus on the global financial and economic system, we focus on the determinants of $\mathrm{G} 7$ and Chinese stock market returns during the COVID-19 outbreak. Our results reveal that Bitcoin and Ethereum can generate benefits from portfolio diversification and hedging strategies for all G7 investors except that of Japan. Monero and Ripple can be considered as diversifier assets for French, Italian, German, and English financial investors during the COVID-19 outbreak. However, the yellow metal is neither hedge nor safe haven during the COVID-19 pandemic. The results indicated that the expected volatility of the US stock market has no effect on the Japanese and Chinese financial markets. Finally, our results suggest that the growth rate of confirmed COVID-19 cases and deaths has no influence on stock market returns except the US market.

\section{REFERENCES}

Aslanidis N., Bariviera A-F., \& Martínez-Ibañez O., (2019). An analysis of cryptocurrencies conditional cross correlations, Finance Research Letters, 31, 130-137.

Baur, D.G., Lucey, B.M., (2010). Is gold a hedge or a safe haven? An analysis of stocks, bonds and gold. The Financial. Review. 45 (2), 217-229.

Beckmann, J., Berger, T., \& Czdaj, R., (2015). Does Gold Act As a Hedge or a Safe Haven for Stocks? A Smooth Transition Approach, Economic Modelling, 48, 16-24.

Bouri, E., Hagfors, L.I., \& Molnar, P., (2017). On the hedge and safe haven properties of Bitcoin: is it really more than a diversifier? Finance Research Letters. 20, 192-198 
Bouri, E, Shahzad, J., Roubaud, D., Kristoufek, L.; \& Lucey, B., (2020). Bitcoin, gold, and commodities as safe havens for stocks: New insight through wavelet analysis, The Quarterly Review of Economics and Finance, doi.org/10.1016/j.qref.2020.03.004.

Brière, M., Oosterlinck, K., \& Szafarz, A., (2015). Virtual currency, tangible return: portfolio diversification with bitcoin. Journal of Asset Management. 16 (6), 365-373

Charfeddine L., Benlagha N., \& Maouchi Y., (2020). Investigating the dynamic relationship between cryptocurrencies and conventional assets: Implications for financial investors. Economic Modelling, $85,198-217$

Corbet, S., Lucey, B., Urquhart, A., \& Yarovaya, L. (2019). Cryptocurrencies as a Financial Asset: A systematic analysis. International Review of Financial Analysis, 62, 182-199

Corbet, S., Meegan, A., Larkin, C., Lucey, B., \& Yarovaya, L., (2018). Exploring the dynamic relationships between cryptocurrencies and other financial assets. Economics letters. 165, 28-34

Dyhrberg, A.H., (2016). Hedging capabilities of Bitcoin. Is it the virtual gold? Finance Research Letters. 16, 139-144

Guesmi, K., Saadi, S., Abid, I., \& Ftiti, Z., (2019). Portfolio diversification with virtual currency: evidence from bitcoin. International Review of Financial Analysis., 63, 431-437.

He, H., Chen, S., Yao, S., \& Ou, J., (2014). Financial liberalisation and international market interdependence: Evidence from China's stock market in the post-WTO accession period. Journal of International Financial Markets, Institutions and Money, 33, 434-444.

International Monetary Fund (2020). Communique of the Forty First Meeting of the IMFC. Retrieved September 28, 2020, from https://www.imf.org/en/News/Articles/2020/04/16/communique-of-theforty-first-meeting-of-the-imfc

Kajtazi, A., Moro, A., (2018). The role of bitcoin in well diversified portfolios: a comparative global study. International Review of Financial Analysis. 2011, 1-15,

Klein, T., Pham Thu, H., \& Walther, T., (2018). Bitcoin is not the New Gold a comparison of volatility, correlation, and portfolio performance. International Review of Financial Analysis. 59, 105-116

Nakamoto, S. (2008). Bitcoin: A Peer-to-Peer Electronic Cash System.

Tiwari A-K. Raheem I-D, \& Kang S-H., (2019). Time-varying dynamic conditional correlation between stock and cryptocurrency markets using the copula-ADCC-EGARCH model. Physica A: 535, 1-9.

Yu, H., Fang, L., Sun, B., \& Du, D. (2018). Risk contribution of the Chinese stock market to developed markets in the post-crisis period. Emerging Markets Review, 34, 87-97. 